\title{
Hamiltonians generating optimal-speed evolutions
}

\author{
Ali Mostafazadeh* \\ Department of Mathematics, Koç University, Sariyer 34450, Istanbul, Turkey
}

(Received 18 April 2008; published 6 January 2009)

\begin{abstract}
We present a simple derivation of the formula for the Hamiltonian operator(s) that achieve the fastest possible unitary evolution between given initial and final states. We discuss how this formula is modified in pseudo-Hermitian quantum mechanics and provide an explicit expression for the most general optimal-speed quasi-Hermitian Hamiltonian. Our approach allows for an explicit description of the metric (inner product) dependence of the lower bound on the travel time and the universality (metric independence) of the upper bound on the speed of unitary evolutions.
\end{abstract}

DOI: 10.1103/PhysRevA.79.014101

PACS number(s): 03.65.Xp, 03.67.Lx, 02.30.Yy, 02.40.-k

In quantum mechanics the travel time for unitary time evolutions between initial and final states $\lambda_{I}$ and $\lambda_{F}$ has a minimum that is proportional to the distance between $\lambda_{I}$ and $\lambda_{F}$ in the state space [1]. For a system with a fixed energy scale, this implies that the speed of unitary evolutions has an upper bound. The problem of determining a Hamiltonian operator that achieves the highest evolution speed has been considered in [2-6]. The purpose of the present paper is to offer a very simple derivation of the formula for a timeindependent optimal-speed Hamiltonian that can be directly generalized to the pseudo-Hermitian representation of quantum mechanics [7]. In particular, we give the explicit form of the most general time-independent quasi-Hermitian [8] optimal-speed Hamiltonian that evolves $\lambda_{I}$ into $\lambda_{F}$.

In the standard formulation of quantum mechanics, the (pure) states of a physical system are identified with the rays in a complex separable Hilbert space $\mathcal{H}$. Each ray can be determined in terms of an associated state vector $\psi \in \mathcal{H}$ $-\{0\}$ according to $\lambda_{\psi}=\{c \psi \mid c \in \mathrm{C}\}$. It is usually convenient to use the one-to-one correspondence between the states $\lambda_{\psi}$ and the projection operators $\Lambda_{\psi}:=\frac{|\psi\rangle\langle\psi|}{\langle\psi \mid \psi\rangle}$ to describe the properties of the space of all states, i.e., the projective Hilbert space $\mathcal{P}(\mathcal{H})$. For a Hilbert space $\mathcal{H}$ of dimension $N \leqslant \infty, \mathcal{P}(\mathcal{H})$ is the complex projective space $\mathrm{C} P^{N-1}$ that plays a central role in the description of geometric phases [9].

It is an easy exercise to show that $\Lambda_{\psi}$ satisfies

$$
\Lambda_{\psi}^{2}=\Lambda_{\psi}=\Lambda_{\psi}^{\dagger}, \quad \operatorname{tr}\left(\Lambda_{\psi}\right)=1
$$

where "tr" denotes the trace. Recall that for a linear operator $L$ acting in $\mathcal{H}, \operatorname{tr}(L):=\sum_{n=1}^{N}\left\langle\xi_{n} \mid L \xi_{n}\right\rangle$, where $\left\{\xi_{n}\right\}$ is an arbitrary orthonormal basis of $\mathcal{H}$ [20].

In view of $(1), \Lambda_{\psi}$ is an element of the space $\mathfrak{B}_{2}(\mathcal{H})$ of all linear operators $L: \mathcal{H} \rightarrow \mathcal{H}$ fulfilling $\operatorname{tr}\left(L^{\dagger} L\right)<\infty$. We can use "tr" to define the following inner product on this space $(L \mid J):=\operatorname{tr}\left(L^{\dagger} J\right)$. This makes $\mathfrak{B}_{2}(\mathcal{H})$ into a separable Hilbert space known as the Hilbert-Schmidt class [10]. Because the state space $\mathcal{P}(\mathcal{H})$ is a subset of $\mathfrak{B}_{2}(\mathcal{H})$, we can use the inner product $(\cdot \mid \cdot)$ to define a notion of distance (metric) on $\mathcal{P}(\mathcal{H})$. We define the line element $d s$ on $\mathcal{P}(\mathcal{H})$ according to

\footnotetext{
*amostafazadeh@ku.edu.tr
}

$$
d s^{2}:=\frac{1}{2}\left(d \Lambda_{\psi \mid} \mid d \Lambda_{\psi}\right)=\frac{\langle\psi \mid \psi\rangle\langle d \psi \mid d \psi\rangle-|\langle\psi \mid d \psi\rangle|^{2}}{\langle\psi \mid \psi\rangle^{2}},
$$

where we have used $\Lambda_{\psi}:=\frac{|\psi\rangle\langle\psi|}{\langle\psi \mid \psi\rangle}$ and (1) [11]. For $N<\infty$ where $\psi$ can be represented by a complex column vector $\overrightarrow{\mathfrak{z}}$ with components $\mathfrak{z}_{1}, \mathfrak{z}_{2}, \ldots, \mathfrak{z}_{N}$, (2) takes the form $d s^{2}$ $=\sum_{a, b=1}^{N} g_{a b *} d \mathfrak{z}_{a} d \mathfrak{z}_{b}^{*}$ where $g_{a b^{*}}:=|\overrightarrow{\mathfrak{z}}|^{-4}\left(|\overrightarrow{\mathfrak{z}}|^{2} \delta_{a b}-\mathfrak{z}_{a}^{*} \mathfrak{z}_{b}\right)$. This is precisely the Fubini-Study metric tensor [12]. For $N=2$, endowing $\mathcal{P}(\mathcal{H})$ with this metric yields a round twodimensional sphere of unit diameter.

Now, suppose that we wish to use an arbitrary Hermitian (self-adjoint) Hamiltonian operator $H: \mathcal{H} \rightarrow \mathcal{H}$ to evolve an initial state $\lambda_{\psi_{I}}$ to a final state $\lambda_{\psi_{F}}$. We can view the evolving state $\lambda_{\psi(t)}$ as a point moving on $\mathcal{P}(\mathcal{H})$. According to (2), the instantaneous speed of the evolution is given by

$$
\frac{d s}{d t}=\frac{\sqrt{\langle\psi(t) \mid \psi(t)\rangle\langle\dot{\psi}(t) \mid \dot{\psi}(t)\rangle-|\langle\psi(t) \mid \dot{\psi}(t)\rangle|^{2}}}{\langle\psi(t) \mid \psi(t)\rangle}=\frac{\Delta E_{\psi(t)}}{\hbar},
$$

where

$$
\Delta E_{\psi(t)}:=\sqrt{\frac{\left\langle\psi(t) \mid H^{2} \psi(t)\right\rangle}{\langle\psi(t) \mid \psi(t)\rangle}-\frac{|\langle\psi(t) \mid H \psi(t)\rangle|^{2}}{\langle\psi(t) \mid \psi(t)\rangle^{2}}}
$$

is the uncertainty in energy and we have employed the Schrödinger equation, $H \psi(t)=i \hbar \dot{\psi}(t)$. We can integrate $d s / d t$ to obtain the length of the curve traced by $\lambda_{\psi(t)}$ in $\mathcal{P}(\mathcal{H})$ as a function of the travel time $\tau[1]$,

$$
s=\frac{1}{\hbar} \int_{0}^{\tau} \Delta E_{\psi(t)} d t
$$

Because $\Delta E_{\psi(t)} \geqslant 0$ for all $t \in[0, \tau), s$ is a monotonically increasing function of $\tau$. This makes $\tau$ a monotonically increasing function of $s$. Therefore, the shortest travel time is achieved for the paths of the shortest length, i.e., the geodesics on $\mathcal{P}(\mathcal{H})[1]$.

Note that the geodesic distance is uniquely determined by the initial and final states and is insensitive to the choice of the Hamiltonian one uses to evolve the initial state along such a geodesic. The travel time depends on the Hamiltonian through the energy uncertainty $\Delta E_{\psi(t)}$. In particular, if one can make the latter arbitrarily large, the travel time can be made arbitrarily small. In typical situations, however, $\Delta E_{\psi(t)}$ 
has a constant upper bound. For example, consider the case that the Hilbert space is finite dimensional $(N<\infty)$ and the energy eigenvalues $E_{n}$ are bounded functions of time, i.e., there is some $\mathcal{E} \in \mathrm{R}^{+}$such $\left|E_{n}(t)\right| \leqslant \mathcal{E}$ for all $n$ and $t$. Then, we can easily show that $\Delta E_{\psi(t)} \leqslant \mathcal{E}$; the travel speed is bounded by $\mathcal{E} / \hbar$; and the travel time has $\hbar s / \mathcal{E}$ as a lower bound. Here $s$ is to be identified with the geodesic distance between the initial and final states.

The above argument is valid, if one does not have additional restrictions on the choice of the Hamiltonian. In practice, one may have to impose constraints that would make it impossible to evolve the initial state along the shortest geodesic connecting it to the final state. In this case one can formulate the problem as a constrained variational problem [5]. In the remainder of this paper we consider constant unconstrained Hamiltonians where the minimum travel time depends, besides the geodesic distance between the initial and final states, on a single real parameter specifying the energy scale of the system.

Let $H$ be a time-independent Hamiltonian operator. Then the time-evolution operator $e^{-i t H / \hbar}$ commutes with $H$ and $H^{2}$, and $\Delta E_{\psi(t)}$ does not depend on $t$. In this case, (4) implies $\tau$ $=\hbar s / \Delta E_{\psi}$, and the speed of the evolution is given by $\Delta E_{\psi} / \hbar$. Therefore, to achieve the highest speed we need to choose the Hamiltonian so that $\Delta E_{\psi} / \hbar$ is maximized. This shows that the travel time is bounded by the ratio of the minimum of $s$, i.e., the geodesic distance between $\lambda_{\psi_{I}}$ and $\lambda_{\psi_{F}}$, to the maximum of speed $\Delta E_{\psi} / \hbar$.

Because we require the evolving state $\lambda_{\psi(t)}$ to trace a geodesic in $\mathcal{P}(\mathcal{H})$ that connects $\lambda_{\psi_{I}}$ and $\lambda_{\psi_{F}}$, it lies entirely in the projective Hilbert space $\mathcal{P}\left(\mathcal{H}^{\prime}\right)$ where $\mathcal{H}^{\prime}$ is the subspace of $\mathcal{H}$ spanned by $\psi_{I}$ and $\psi_{F}$. This is in fact a characteristic property of the Fubini-Study metric $[1,13]$. It shows that we can restrict our attention to the case that $\mathcal{H}$ is two dimensional; $N=2$ [4]. Furthermore, without loss of generality, we can suppose that $\operatorname{tr}(H)=0$. This implies that the eigenvalues of $H$ have opposite sign, $E_{2}=-E_{1}=: E$. Let $\left\{\psi_{1}, \psi_{2}\right\}$ be an orthonormal basis consisting of the eigenvectors of $H, H \psi_{n}$ $=E_{n} \psi_{n}$. We expand $\psi(0)=\psi_{I}$ in this basis to find

$$
\psi_{I}=c_{1} \psi_{1}+c_{2} \psi_{2}, \quad c_{1}, c_{2} \in \mathrm{C},
$$

and use the time independence of $\Delta E_{\psi}$ to compute it at $t$ $=0$. In view of (3) and (5), this yields

$$
\Delta E_{\psi}=E \sqrt{1-\left(\frac{\left|c_{1}\right|^{2}-\left|c_{2}\right|^{2}}{\left|c_{1}\right|^{2}+\left|c_{2}\right|^{2}}\right)^{2}} \leqslant E .
$$

Therefore, the travel time $\tau$ satisfies

$$
\tau \geqslant \tau_{\min }:=\frac{\hbar s}{E},
$$

where $s$ is the geodesic distance between $\lambda_{\psi_{I}}$ and $\lambda_{\psi_{F}}$ in $\mathcal{P}(\mathcal{H})$. Equation (7) identifies $\tau_{\min }$ with a lower bound on the travel time. Next, we construct a Hamiltonian $H_{\star}$ with eigenvalues $\pm E$ for which $\tau=\tau_{\min }$. This shows that indeed $\tau_{\min }$ is the minimum travel time.
Because $s$ is completely determined by $\lambda_{\psi_{I}}$ and $\lambda_{\psi_{F}}$, the condition $\tau=\tau_{\min }$ is fulfilled if and only if $\Delta E_{\psi}=E$. In light of (6) this is equivalent to $\left|c_{1}\right|=\left|c_{2}\right|$. If we expand $\psi_{F}$ in the basis $\left\{\psi_{1}, \psi_{2}\right\}$ to find

$$
\psi_{F}=d_{1} \psi_{1}+d_{2} \psi_{2}, \quad d_{1}, d_{2} \in \mathbb{C},
$$

and compute $\Delta E_{\psi}$ at $t=\tau$, we obtain (6) with $\left(c_{1}, c_{2}\right)$ replaced with $\left(d_{1}, d_{2}\right)$. As a result, in order to maintain $\Delta E_{\psi}=E$, we must have $\left|d_{1}\right|=\left|d_{2}\right|$.

Next we express $\left|c_{1}\right|=\left|c_{2}\right|$ and $\left|d_{1}\right|=\left|d_{2}\right|$ in the form $c_{2}$ $=e^{i \alpha_{I}} c_{1}$ and $d_{2}=e^{i \alpha_{F}} d_{1}$, for some $\alpha_{I}, \alpha_{F} \in \mathbb{R}$, respectively. Substituting these in (5) and (8), we find

$$
\psi_{1}+e^{i \alpha_{I}} \psi_{2}=c_{1}^{-1} \psi_{I}, \quad \psi_{1}+e^{i \alpha_{F}} \psi_{2}=d_{1}^{-1} \psi_{F} .
$$

We can solve these equations for $\psi_{1}$ and $\psi_{2}$ in terms of $\psi_{I}$ and $\psi_{F}$, and use the spectral resolution of $H_{\star}$, i.e.,

$$
H_{\star}=E\left(-\left|\psi_{1}\right\rangle\left\langle\psi_{1}|+| \psi_{2}\right\rangle\left\langle\psi_{2}\right|\right),
$$

to compute $H_{\star}$. This calculation is more conveniently performed in terms of

$$
\vartheta:=\alpha_{I}-\alpha_{F}, \quad \hat{\psi}_{I}:=\frac{\psi_{I}}{\sqrt{2} c_{1}}, \quad \hat{\psi}_{F}:=\frac{e^{i \vartheta / 2} \psi_{F}}{\sqrt{2} d_{1}} .
$$

The result is $[5,6]$

$$
\begin{aligned}
H_{\star} & =\frac{i E\left(\left|\hat{\psi}_{F}\right\rangle\left\langle\hat{\psi}_{I}|-| \hat{\psi}_{I}\right\rangle\left\langle\hat{\psi}_{F}\right|\right)}{4 \sin \left(\frac{\vartheta}{2}\right)} \\
& =\frac{i E \cot \left(\frac{\vartheta}{2}\right)}{4}\left(\frac{\left|\psi_{F}\right\rangle\left\langle\psi_{I}\right|}{\left\langle\psi_{I} \mid \psi_{F}\right\rangle}-\frac{\left|\psi_{I}\right\rangle\left\langle\psi_{F}\right|}{\left\langle\psi_{F} \mid \psi_{I}\right\rangle}\right),
\end{aligned}
$$

where we have used the fact that $\hat{\psi}_{I}$ and $\hat{\psi}_{F}$ are unit vectors. Moreover, (11) implies $\cos ^{2}(\vartheta / 2)=\frac{\left|\left\langle\psi_{l} \mid \psi_{F}\right\rangle\right|^{2}}{\left\langle\psi_{I} \mid \psi_{I}\right\rangle\left\langle\psi_{F} \mid \psi_{F}\right\rangle}$, which as explained in [1] identifies $\vartheta$ with $2 s$ [21].

Equation (13) can be easily modified to give the expression for the optimal-speed Hamiltonians in pseudoHermitian quantum mechanics. One merely needs to make the following substitution in the above analysis:

$$
\left|\psi_{n}\right\rangle \rightarrow\left|\psi_{n}\right\rangle, \quad\left\langle\psi_{n}\right| \rightarrow\left\langle\psi_{n}\right|:=\left\langle\psi_{n}\right| \eta_{+}, \quad s \rightarrow s_{\eta_{+}},
$$

where $\eta_{+}: \mathcal{H} \rightarrow \mathcal{H}$ is the metric operator that defines the inner product of the physical Hilbert space $\mathcal{H}_{\text {phys }}$, i.e., $\langle\cdot, \cdot\rangle_{\eta_{+}}:=\left\langle\cdot \mid \eta_{+} \cdot\right\rangle=\langle\cdot \mid \cdot\rangle$, and $s_{\eta_{+}}$is the distance defined by the natural metric on the projective Hilbert space $\mathcal{P}\left(\mathcal{H}_{\text {phys }}\right)$. We can obtain the line element associated with this metric by making the substitutions (14) on the right-hand side of (2). This gives [11]

$$
d s_{\eta_{+}}^{2}:=\frac{\langle\psi \mid \psi\rangle\left\langle d \psi|d \psi>-|<\psi|d \psi>|^{2}\right.}{<\psi \mid \psi>^{2}} .
$$

Similarly we find the following expressions for minimum travel time $\tau_{\text {min }}^{\left(\eta_{+}\right)}$and the optimal-speed $\eta_{+}$-pseudo-Hermitian [14] Hamiltonian $H_{\star}^{\left(\eta_{+}\right)}$(with eigenvalues $\pm E$ ), 


$$
\begin{gathered}
\tau_{\min }^{\left(\eta_{+}\right)}=\frac{\hbar s_{\eta_{+}}}{E}, \\
H_{\star}^{\left(\eta_{+}\right)}=\frac{i E \cot \left(s_{\eta_{+}}\right)}{4}\left(\frac{\left|\psi_{F}><\psi_{I}\right|}{\left\langle\psi_{I} \mid \psi_{F}\right\rangle}-\frac{\left|\psi_{I}><\psi_{F}\right|}{\left\langle\psi_{F} \mid \psi_{I}\right\rangle}\right),
\end{gathered}
$$

where

$$
\cos ^{2}\left(s_{\eta_{+}}\right)=\frac{\left|<\psi_{I}\right| \psi_{F}>\left.\right|^{2}}{<\psi_{I}\left|\psi_{I}><\psi_{F}\right| \psi_{F}>} .
$$

According to (7) and (16), if we choose $\eta_{+}$such that the geodesic distance $s_{\eta_{+}}$between $\lambda_{\psi_{I}}$ and $\lambda_{\psi_{F}}$ in $\mathcal{P}\left(\mathcal{H}_{\text {phys }}\right)$ is smaller than the geodesic distance $s$ between $\lambda_{\psi_{I}}$ and $\lambda_{\psi_{F}}$ in $\mathcal{P}(\mathcal{H})$, then $\tau_{\text {min }}^{\left(\eta_{+}\right)}<\tau_{\text {min }}$. This is the essence of the main result of [15]. Indeed, as we show below, it is possible to choose $\eta_{+}$ so that regardless of the choice of $\lambda_{\psi_{I}}$ and $\lambda_{\psi_{F}}$ their distance in $\mathcal{P}\left(\mathcal{H}_{\text {phys }}\right)$ becomes arbitrarily small. But this does not seem to have any physical implications, for such an evolution amounts to evolving a state to an arbitrarily close state in an arbitrarily short time. The physical quantity of practical significance, particularly in areas such as quantum computation, is the speed of the evolution, namely $E / \hbar$, which is a universal quantity independent of the choice of $\eta_{+}$. Therefore, the minimum travel time between states of a given distance is independent of $\eta_{+}$[11]. A physical process that involves evolving $\lambda_{\psi_{I}}$ into $\lambda_{\psi_{F}}$ in $\mathcal{P}\left(\mathcal{H}_{\text {phys }}\right)$ using an $\eta_{+}$-pseudo-Hermitian Hamiltonian $H: \mathcal{H} \rightarrow \mathcal{H}$ in time $\tau$ may be described equally well by evolving $\lambda_{\eta_{+}^{1 / 2} \psi_{I}}$ into $\lambda_{\eta_{+}^{1 / 2} \psi_{F}}$ in $\mathcal{P}(\mathcal{H})$ using the equivalent Hermitian Hamiltonian $h$ $:=\eta_{+}^{1 / 2} H \eta_{+}^{-1 / 2}$ in the same time $\tau$. As shown in [11], the length of the curve corresponding to these evolutions in the respective projective Hilbert spaces are identical. Therefore, they will have the same speed.

Next, we wish to show how by choosing the metric operator we may adjust the value of $s_{\eta_{+}}$and consequently $\tau_{\min }^{\left(\eta_{+}\right)}$. Again, without loss of generality we confine our attention to the case $N=2$. Let $\left\{e_{1}, e_{2}\right\}$ denote the standard basis of $\mathrm{C}^{2}$, i.e., $e_{1}:=\left(\begin{array}{l}1 \\ 0\end{array}\right), e_{2}:=\left(\begin{array}{l}0 \\ 1\end{array}\right)$. Then we can represent any metric operator $\eta_{+}$in $\left\{e_{1}, e_{2}\right\}$ by a positive-definite matrix of the form

$$
\underline{\eta_{+}}=\left(\begin{array}{cc}
a & \mathfrak{b}^{*} \\
\mathfrak{b} & c
\end{array}\right)
$$

where $a, c \in \mathbb{R}$ and $\mathfrak{b} \in \mathbb{C}$. Because $\eta_{+}$is a positive-definite matrix,

$$
a+c=\operatorname{tr}\left(\underline{\eta_{+}}\right)>0, \quad D:=a c-|\mathfrak{b}|^{2}=\operatorname{det}\left(\underline{\eta_{+}}\right)>0 .
$$

For states $\lambda_{\psi}$, differing from $\lambda_{e_{2}}$, we can use a representative state vector of the form $\psi:=\left(\begin{array}{c}1 \\ x+i y\end{array}\right)$. Substituting this relation in (15) and using (14) and (19) we find [11]

$$
d s_{\eta_{+}}^{2}=\frac{D\left(d x^{2}+d y^{2}\right)}{\left[a+2\left(b_{1} x+b_{2} y\right)+c\left(x^{2}+y^{2}\right)\right]^{2}},
$$

where $b_{1}$ and $b_{2}$ are, respectively, the real and imaginary parts of $\mathfrak{b}$, i.e., $\mathfrak{b}=: b_{1}+i b_{2}$.

Next, we introduce the angular coordinates $(\varphi, \theta)$ that are related to $(x, y)$ according to $x=\tan \left(\frac{\theta}{2}\right) \cos (\varphi+\beta), \quad y$ $=\tan \left(\frac{\theta}{2}\right) \sin (\varphi+\beta)$, where $\beta:=\tan ^{-1}\left(b_{2} / b_{1}\right)$. These coordinates also allow for treating the state $\lambda_{e_{2}}$. To see this, first observe that for $(x, y) \in \mathbb{R}^{2}$, we have $\varphi \in[0,2 \pi)$ and $\theta$ $\in[0, \pi)$. The state $\lambda_{e_{2}}$ corresponds to the point at infinity in the $x-y$ plane which we can identify with $\theta=\pi$. In terms of $(\varphi, \theta),(21)$ reads as

$$
d s_{\eta_{+}}^{2}=\frac{k_{1}\left(d \theta^{2}+\sin ^{2} \theta d \varphi^{2}\right)}{\left(1+k_{2} \cos \theta+k_{3} \cos \varphi \sin \theta\right)^{2}},
$$

where we have introduced $k_{1}:=\frac{D}{(a+c)^{2}}=\frac{\operatorname{det}\left(\underline{\left.\eta_{+}\right)}\right.}{\operatorname{tr}\left(\underline{\eta_{+}}\right)^{2}}, k_{2}:=\frac{a-c}{a+c}$, and $k_{3}:=\frac{2|\mathfrak{b}|}{a+c}$. Note that because of $(20)$ we have $k_{1}>0,-1<k_{2}$ $<1$, and $0 \leqslant k_{3}<1$.

If we set $\eta_{+}=I$ we recover the standard Euclidean inner product on the Hilbert space. In this case $s^{\eta_{+}}=s, a=c=1, \mathfrak{b}$ $=0, \quad k_{1}=1 / 4, \quad k_{2}=k_{3}=0$, and (22) becomes $d s^{2}=\frac{1}{4}\left(d \theta^{2}\right.$ $\left.+\sin ^{2} \theta d \varphi^{2}\right)$. This is just the standard metric for a round sphere of unit diameter. In [11] we show that $\mathcal{P}\left(\mathcal{H}_{\text {phys }}\right)$ is related to $\mathcal{P}(\mathcal{H})$ by an isometry. Therefore, (22) also describes a round sphere of unit diameter, and $(\varphi, \theta)$ are the usual spherical coordinates.

We can use (17) and (19) to obtain the explicit form of the optimal-speed $\eta_{+}$-pseudo-Hermitian Hamiltonians for given initial $\lambda_{\psi_{I}}$ and final $\lambda_{\psi_{F}}$ states. We can always perform an invertible linear (basis) transformation in $\mathcal{H}$ so that $\lambda_{\psi_{I}}=\lambda_{e_{1}}$. Let $\lambda_{\psi_{F}}$ be an arbitrary final state (that is different from $\lambda_{e_{1}}$ ). Then we can take $\psi_{I}=\left(\begin{array}{l}1 \\ 0\end{array}\right)$, and $\psi_{F}=\left(\begin{array}{l}\zeta \\ 1\end{array}\right)$, for some $\zeta \in \mathbb{C}$. In view of (14) and (19), we have $\left\langle\psi_{I} \mid \psi_{I}\right\rangle=a,\left\langle\psi_{F} \mid \psi_{F}\right\rangle$ $=\frac{D+|\xi|^{2}}{a},\left\langle\psi_{I}\right| \psi_{F}>=a \zeta+\mathfrak{b}^{*}=: \xi$. Inserting these in (18) and using (16), we find $\cos s_{\eta_{+}}=|\xi| / \sqrt{D+|\xi|^{2}}$ and

$$
\tau_{\text {min }}^{\left(\eta_{+}\right)}=\frac{\hbar}{E} \cos ^{-1}\left(|\xi| / \sqrt{D+|\xi|^{2}}\right) .
$$

Similarly we employ (17) and (19) to obtain the matrix representation of $H_{\star}^{\left(\eta_{+}\right)}$in the basis $\left\{e_{1}, e_{2}\right\}$,

$$
\underline{H_{\star}}{ }^{\left(\eta_{+}\right)}=\frac{i E e^{-i \omega}}{4 a \sqrt{D}}\left(\begin{array}{cc}
-a \mathfrak{b}^{*} & -\left(D e^{2 i \omega}+\mathfrak{b}^{* 2}\right) \\
a^{2} & a \mathfrak{b}^{*}
\end{array}\right),
$$

where $\omega:=\arg (\xi)$, i.e., $e^{i \omega}=\xi /|\xi|$. It is interesting to see that the minimum travel time and optimal-speed Hamiltonians are, respectively, determined by the modulus and the phase of $\xi$. Note also that the right-hand side of (24) does not have a unique limit as $\xi \rightarrow 0$. This is because $\xi=0$ corresponds to the case that $\lambda_{\psi_{I}}$ and $\lambda_{\psi_{F}}$ are antipodal points of $\mathcal{P}\left(\mathcal{H}_{\text {phys }}\right)$ that are connected via an infinity of geodesics with equal length.

Setting $a=c=D=1$ and $\mathfrak{b}=0$ in (23) and (24), we find the explicit form of the optimal-speed Hamiltonian and the minimum travel time in conventional quantum mechanics:

$$
\begin{gathered}
\underline{H_{\star}}=\frac{i E}{4}\left(\begin{array}{cc}
0 & -e^{i \omega} \\
e^{-i \omega} & 0
\end{array}\right), \\
\tau_{\min }=\frac{\hbar}{E} \cos ^{-1}\left(|\zeta| / \sqrt{1+|\zeta|^{2}}\right) .
\end{gathered}
$$

Note that in this case $\xi=\zeta$ and $e^{i \omega}=\zeta /|\zeta|$. Comparing (25) with (23), we see that by keeping $a$ and $\mathfrak{b}$ fixed, so that $\xi$ is 
left unchanged, and decreasing the value of $c$ we can make $D$ as small as we wish. This in turn reduces the value of $\tau_{\text {min }}^{\left(\eta_{+}\right)}$ below that of $\tau_{\min }$. For example, we can set $a=1$ and $\mathfrak{b}=0$. Then, $D=c, \xi=\zeta$, and we find

$$
{\underline{H_{\star}}}^{\left(\eta_{+}\right)}=\frac{i E}{4}\left(\begin{array}{cc}
0 & -\sqrt{c} e^{i \omega} \\
\frac{e^{-i \omega}}{\sqrt{c}} & 0
\end{array}\right),
$$

and $\tau_{\min }^{\left(\eta_{+}\right)}=\frac{\hbar}{E} \cos ^{-1}\left(|\zeta| / \sqrt{c+|\zeta|^{2}}\right)$. For $c<1$, this yields $\tau_{\min }^{\left(\eta_{+}\right)}$ $<\tau_{\min }$. As we explained above, this observation does not seem to have any practical implications, if we use $H_{\star}^{\left(\eta_{+}\right)}$to generate a unitary evolution, i.e., consider the dynamics taking place in $\mathcal{P}\left(\mathcal{H}_{\text {phys }}\right)$. If we instead consider the dynamics defined by $H_{\star}^{\left(\eta_{+}\right)}$in $\mathcal{P}(\mathcal{H})$, then the travel time is still given by (23) (which can be made smaller than $\tau_{\min }$ ) but the evolution is nonunitary. This is a manifestation of the nonexistence of an upper bound on evolution speed for nonunitary evolutions [16]. A more interesting observation is that one can realize this fact using a quasi-Hermitian Hamiltonian $(\mathcal{P} \mathcal{T}$-symmetric Hamiltonians considered in [15] being special cases) [17]; there is no upper bound on the speed of quasiunitary evolutions [22].

To offer a physical interpretation for this result we first recall that in quantum mechanics, a physical system is represented by a Hilbert space-Hamiltonian pair $(\mathcal{H}, H)$. This representation is, however, not unique, for unitary-equivalent Hilbert space-Hamiltonian pairs describe the same system. If
$\mathcal{H}$ and $\mathcal{H}_{\text {phys }}$ are Hilbert spaces that have identical vector space structure but different inner products (say corresponding to the choices $I$ and $\eta_{+}$for their metric operators, respectively), one can use a single Hamiltonian operator to represent two different quantum systems, e.g., $\left(\mathcal{H}, H_{\star}^{\left(\eta_{+}\right)}\right)$and $\left(\mathcal{H}_{\text {phys }}, H_{\star}^{\left(\eta_{+}\right)}\right)$represent distinct physical systems with quasiunitary and unitary dynamical evolutions, respectively. The above argument shows that while the evolution speed for the latter system is given by $E / \hbar$, that of the former can be made arbitrarily large. To determine whether this observation can have practical applications requires a more detailed investigation of the role of quasi- and pseudo-Hermitian Hamiltonians in open quantum systems [18].

In summary, we have offered a straightforward derivation of an expression for the most general time-independent optimal-speed quasi-Hermitian (in particular Hermitian) Hamiltonians and established by explicit calculation the metric dependence of the minimum travel time and metric independence of the maximum travel speed. Our analysis confirms the existence of infinitely fast quasiunitary evolutions. These might find applications in areas such as quantum computation and quantum control $[3,19]$. The derivation of an explicit expression for the most general optimal-speed quasiHermitian Hamiltonian, which we have reported here, is a necessary step in this direction.

This project was supported by grants provided by TÜBİTAK (the Scientific and Technological Research Council of Turkey) and TÜBA (Turkish Academy of Sciences).
[1] J. Anandan and Y. Aharonov, Phys. Rev. Lett. 65, 1697 (1990).

[2] G. N. Fleming, Nuovo Cimento Soc. Ital. Fis., A 16, 232 (1973); L. Vaidman, Am. J. Phys. 60, 182 (1992).

[3] N. Margolus and L. B. Levitin, Physica D 120, 188 (1998).

[4] D. C. Brody, J. Phys. A 36, 5587 (2003).

[5] A. Carlini, A. Hosoya, T. Koike, and Y. Okudaira, Phys. Rev. Lett. 96, 060503 (2006); Phys. Rev. A 75, 042308 (2007).

[6] D. C. Brody and D. W. Hook, J. Phys. A 39, L167 (2006).

[7] A. Mostafazadeh and A. Batal, J. Phys. A 37, 11645 (2004).

[8] F. G. Scholtz, H. B. Geyer, and F. J. W. Hahne, Ann. Phys. (N.Y.) 213, 74 (1992).

[9] D. N. Page, Phys. Rev. A 36, 3479 (1987); A. Bohm and A. Mostafazadeh, J. Math. Phys. 35, 1463 (1994); A. Bohm, A. Mostafazadeh, H. Koizumi, Q. Niu, and J. Zwanziger, The Geometric Phase in Quantum Systems (Springer, New York, 2003).

[10] M. Reed and B. Simon, Functional Analysis (Academic, San Diego, 1980), Vol. I.

[11] A. Mostafazadeh, Phys. Rev. Lett. 99, 130502 (2007).

[12] T. Eguchi, P. B. Gilkey, and A. J. Hanson, Phys. Rep. 66,
213 (1980).

[13] I. Bengtsson and K. Życzkowski, Geometry of Quantum States (Cambridge University Press, Cambridge, 2006).

[14] A. Mostafazadeh, J. Math. Phys. 43, 205 (2002); 43, 2814 (2002); 43, 3944 (2002).

[15] C. M. Bender, D. C. Brody, H. F. Jones, and B. K. Meister, Phys. Rev. Lett. 98, 040403 (2007).

[16] P. E. G. Assis and A. Fring, J. Phys. A 41, 244002 (2008); A. Mostafazadeh, e-print arXiv:0709.1756.

[17] H. F. Jones (private communication).

[18] M. Jakob and S. Stenholm, Phys. Rev. A 70, 012104 (2004).

[19] N. Khaneja, R. Brockett, and S. J. Glaser, Phys. Rev. A 63, 032308 (2001).

[20] $\operatorname{tr}(L)$ is independent of the choice of $\left\{\xi_{n}\right\}$.

[21] In view of this expression for $\cos ^{2}(\vartheta / 2),(11)$, and $\left\langle\hat{\psi}_{I} \mid \hat{\psi}_{I}\right\rangle$ $=\left\langle\hat{\psi}_{F} \mid \hat{\psi}_{F}\right\rangle=1, \quad c_{1}=\sqrt{\left\langle\psi_{I} \mid \psi_{I}\right\rangle / 2} e^{i \gamma}, \quad$ and $\quad d_{1}=\sqrt{\left\langle\psi_{F} \mid \psi_{F}\right\rangle / 2} e^{i \delta}$, where $\gamma, \delta \in \mathbb{R}$ are arbitrary if $\left\langle\psi_{I} \mid \psi_{F}\right\rangle=0$, otherwise $e^{i(\delta-\gamma)}$ $=e^{i \vartheta / 2}\left\langle\psi_{I} \mid \psi_{F}\right\rangle /\left|\left\langle\psi_{I} \mid \psi_{F}\right\rangle\right|$.

[22] $U: \mathcal{H} \rightarrow \mathcal{H}$ is quasiunitary, if it is $\eta_{+}$-pseudounitary for some metric operator $\eta_{+}$, i.e., $U^{-1}=\eta_{+} U^{\dagger} \eta_{+}^{-1}$. See A. Mostafazadeh, J. Math. Phys. 45, 932 (2004). 